\title{
Segmental Relaxation of Poly(styrene-co-vinylphenol)
}

\author{
M. J . Schroeder \\ Department of Chemistry, United States Naval Academy, Annapolis, Maryland 21402 \\ C. M. Roland* \\ Chemistry Division, Code 6120, Naval Research Laboratory, Washington, D.C. 20375-5342

\section{T. K. Kwei} \\ Department of Chemical Engineering, Chemistry and Material Science, Polytechnic University, \\ Six Metrotech Center, Brooklyn, New York 11201
}

Received March 2, 1999; Revised Manuscript Received J uly 22, 1999

\begin{abstract}
Dielectric and mechanical measurements of the $\alpha$-relaxation are reported for polystyrenes of varying vinylphenol content. The presence of the polar moiety substantially increases both the glass transition temperature and the segmental relaxation times of the polymer. However, the shape of the relaxation function, as well as the temperature dependence of the relaxation times, is invariant to copolymer composition. This indicates that the enhanced interactions arising from polar modification of the polystyrene are short-lived on the time scale of the segmental dynamics. Although fragility was found to be independent of copolymer composition, the heat capacity change at $\mathrm{T}_{\mathrm{g}}$ increases with increasing vinylphenol content. The absence of a correlation between these two quantities is contrary to an energy landscape interpretation of fragility.
\end{abstract}

\section{Introduction}

The segmental dynamics of polymers underlie to some extent motions at all longer times; thus, investigation of the structural relaxation associated with the glass transition has obvious appeal. Theoretical and experimental efforts to understand the $\alpha$-relaxation are longstanding. There is a dichotomy in the direction of this research between a focus on short time (ca. $\leq 10^{-9} \mathrm{~s}$ ) behavior versus characterization of properties at macroscopic time scales at temperatures near the glass transition. The high-frequency behavior of polymers and other glass-forming liquids is complicated by secondary relaxations, Boson peaks, vibrational anharmonicity, etc., which makes interpretation difficult.1,2 At longer times, intermolecular cooperativity dominates, ${ }^{1-6}$ although a connection to the higher frequency dynamics remains.

Copolymers offer an opportunity to explore these issues, allowing structure/property relationships to be probed via systematic changes in chemical composition. ${ }^{7-15}$ In this paper we describe dielectric and mechanical measurements on polystyrene copolymerized with varying levels of vinylphenol. The hydrogenbonding capacity of the latter enhances miscibility, leading to a number of interesting blends. ${ }^{16-22}$ In addition, introduction of the hydroxyl group increases the polarity of the polystyrene. ${ }^{23}$ We expect the consequent increase in intermolecular interactions to directly influence the segmental dynamics.

\section{Experimental Section}

Random copolymers of styrene and 4-vinylphenol were synthesized by copolymerization of styrene and p-acetoxystyrene with AIBN as the initiator. Acetoxy functional groups were subsequently reduced using hydrazine hydrate. Films were cast from acetone or cyclohexane onto the electrodes and then dried in a vacuum at temperatures above $T_{g}$. Vinylphenol contents as high as $40 \mathrm{~mol} \%$ were prepared, with the chemical structure verified by $\mathrm{H}^{1}$ NMR. Due to problems with thermal degradation, dielectric measurements could be reliably obtained only on samples having $18 \%$ vinylphenol .

I sothermal dielectric measurements were obtained using a time domain spectrometer (I mass Inc.) having a frequency range $-3.2<\log \omega(\mathrm{rad} / \mathrm{s})<4.8$. A $25 \mathrm{~mm}$ parallel plate geometry was employed, with a guard ring on the detector side. Samples were 0.1-0.2 mm thick. A Delta Design model 9023 oven provided temperature control to $\pm 0.1 \mathrm{~K}$. Samples were allowed to equilibrate for at least an hour after attaining the desired temperature. Numerous repeat measurements were made to ensure the absence of both solvent and sample degradation.

Dynamical mechanical spectra were obtained with a Bohlin VOR rheometer, again using a parallel plate geometry (sample radii and gap equal to 6 and $0.9 \mathrm{~mm}$, respectively). The dynamic shear modulus was measured from $6 \mathrm{rad} / \mathrm{s}$, down to as low as $10^{-4} \mathrm{rad} / \mathrm{s}$ for the lower temperatures, at strains in the range from 0.1 to $3 \%$.

Differential scanning calorimetry (DSC) was carried out by cooling at $10 \mathrm{deg} / \mathrm{min}$ using a Perkin-Elmer DSC-7. Glass transition temperatures, $\mathrm{T}_{\mathrm{g}}$, were taken to be the temperatures corresponding to half the exothermic shift.

\section{Results and Discussion}

The $\alpha$-dispersion in the dielectric loss was measured for the various polymers, with a representative spectrum shown in Figure 1. For the lower temperatures, electrical conductivity intrudes at lower frequency. This was subtracted from the spectra by fitting to a power $\operatorname{law}^{24}$

$$
\epsilon^{\prime \prime}(\omega)_{\text {cond }}=\sigma \omega^{-\mathrm{s}}
$$

where $\sigma$ and $\mathrm{s}(0.5 \leq \mathrm{s} \leq 1)^{25}$ are adjustable parameters. After removal of $\epsilon^{\prime \prime}(\omega)_{\text {cond }}$, a characteristic time for local segmental relaxation is defined from the frequency of the maximum in the dielectric loss, $\tau_{\alpha} \equiv 1 / \omega_{\max }$. These relaxation times are plotted in Arrhenius fashion in Figure 2 for each sample. The curves through the data 


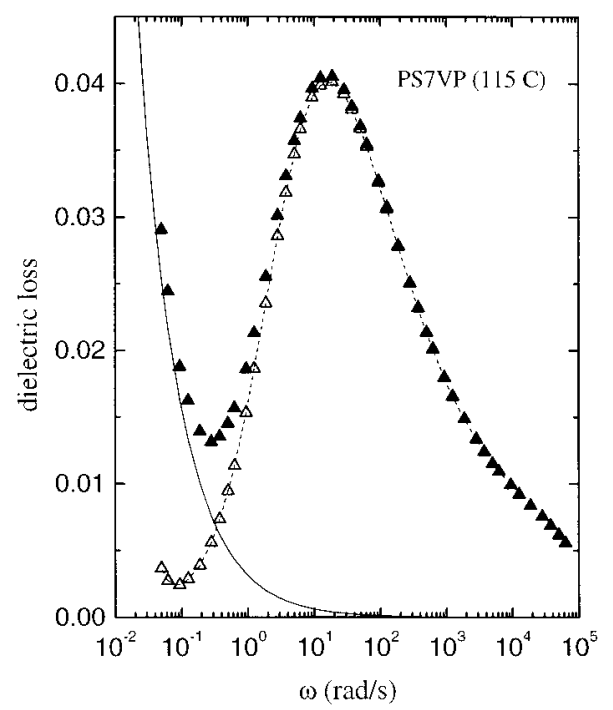

Figure 1. Dielectric loss for PS7VP ( $\mathbf{\Delta})$. The solid line represents the fit to the dc conductivity (eq 1 ), the subtraction of which yields the relaxation function $(\Delta)$. The dotted line is the KWW function (eq 5) with $\beta=0.43$.

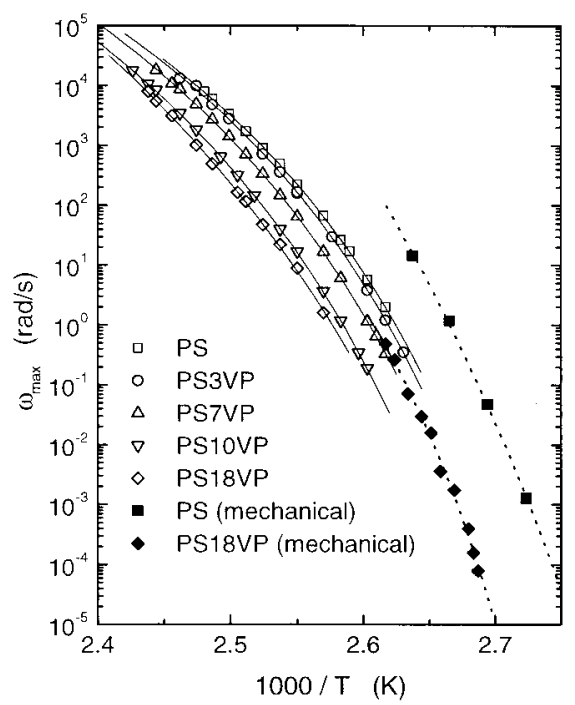

Figure 2. Inverse of the frequency of the maximum in the dielectric loss (hollow symbols) and the loss modulus (solid symbols). The lines are fits to eq 2.

represent fits to the Vogel-Fulcher equation 26

$$
\tau=\tau_{0} \exp _{\overline{\mathrm{T}}-\mathrm{T}_{0}}
$$

with the obtained Vogel-Fulcher parameters listed in Table 1.

Using eq 2 to interpolate the data in Figure 2, we determine the temperature, $T_{\alpha}$, at which the relaxation time equals $1 \mathrm{~s}$. These are shown in Figure 3, along with the glass transition temperatures determined by DSC. There is a rough correspondence between the two quantities; a common "rule of thumb" is that $\mathrm{T}_{\mathrm{g}}$ measured calorimetrically at $10 \mathrm{deg} / \mathrm{min}$ is equal to the temperature at which $\tau=100 \mathrm{~s} \cdot{ }^{27,28}$ However, it is difficult to accurately measure the diel ectric spectrum at very low frequencies $\left(\omega_{\max }=0.01 \mathrm{rad} / \mathrm{s}\right)$, thus our choice of $\tau_{\alpha}=1 \mathrm{~s}$ for $\mathrm{T}_{\alpha}$. Also shown in Figure 3 is the heat capacity change at $T_{g}$ measured for the polymers. Both $\Delta \mathrm{C}_{\mathrm{p}}\left(\mathrm{T}_{\mathrm{g}}\right)$ and the transition temperatures, $\mathrm{T}_{\mathrm{g}}$ and $\mathrm{T}_{\alpha}$, are seen to be increasing functions of the vinyl phenol content.
The dielectric loss spectrum is related to the relaxation function by

$$
\phi(\mathrm{t})=\frac{2}{\pi} \int_{0}^{\infty \epsilon^{\prime \prime}(\omega)} \frac{\cos \omega \mathrm{t}}{\Delta \epsilon} \mathrm{d} \omega
$$

where $\Delta \epsilon$ is the dielectric strength

$$
\Delta \epsilon=\int_{0}^{\infty} \epsilon^{\prime \prime}(\omega) \mathrm{d} \log \omega
$$

Values for $\Delta \epsilon$ are listed in Table 1. One would anticipate the dielectric strength to increase with vinylphenol content, reflecting theincreasing polarity of the polymer chains. ${ }^{23}$ This is the case (ignoring the weak inverse dependence on temperature), ${ }^{29}$ except for the copolymer having the highest vinylphenol. The $\Delta \epsilon$ for PS18VP is lower than for the PS7VP and PS10VP. Such behavior has been seen previously in copolymers ${ }^{30-32}$ and ascribed to dipolar canceling due to the backbone geometry.

Spectra for the samples representing the extremes of vinylphenol content, PS and PS18VP, are shown in Figure 4. The data have been shifted to superimpose the peaks. There is only a very slight broadening of the dispersion in going from zero to the highest vinyl phenol content studied herein. This broadening is reflected in the values of the stretch exponent, $\beta$, for the Kohlrausch-William-Watts (KWW) function ${ }^{33,34}$ describing the dielectric relaxation

$$
\phi(\mathrm{t})=\exp \left(-\frac{\mathrm{t}}{\tau_{\mathrm{K} w w}}\right)^{\beta}
$$

The time for this relaxation function to decrease to its $\mathrm{e}^{-1}$ value, $\tau_{\mathrm{K} w \mathrm{w}}$, is slightly less than $\tau$. A representative fit to eq 5 is included in Figure 4 . The best-fit $\beta$ for all samples are listed in Table 1. For these polymers, $\beta$ does not change significantly over the range of temperatures at which the loss peak could be accurately measured. However, there is a slight decrease in $\beta$ with vinyl phenol content.

The similarity of the shape of the relaxation functions for the different polymers can be illustrated by means of a scaling method, which has been proposed to yield a universal form for the $\alpha$-relaxation function. ${ }^{35-38} \mathrm{Al}$ though the resulting master curves have been criticized as potentially obscuring features that may arise in the spectral wings, ${ }^{39,40}$ the approach suffices herein to demonstrate the similarity of the relaxation behavior of these polymers. Derived from multifractal analysis, the ordinate is defined as $\left(\left(\epsilon^{\prime \prime} \omega_{\max } / \Delta \epsilon \omega\right)\right)^{1 / \mathrm{w}}$, where $\mathrm{w}$ is the full width of the peak at half-maximum on a logarithmic frequency scale, normalized by the Debye width (1.142 decades). The abscissa used in this scaling is $\left(\omega / \omega_{\max }\right)^{\left(1+w^{-1}\right) / w}$. The requisite parameters are listed in Table 1, with w given to a good approximation by $w$ $=1.047 /(\beta+0.047) .{ }^{41}$

As shown in Figure 5, the scaling causes a collapse of the data for all samples, except for some deviation at the lowest frequencies, where the relaxation is obscured to some extent by sample conductivity. Also shown in the figure is eq 5 for $\beta=0.43$. This describes the $\alpha$-relaxation well in the center of the dispersion ( $F$ igure 4) but underestimates the experimental intensities at highest frequencies. It is generally found that dielectric 
Table 1. Dielectric Relaxation Results

\begin{tabular}{llcccccc}
\hline & \multicolumn{1}{c}{ polymer } & $\Delta \epsilon$ & $\beta$ & $\tau_{0}(\mathrm{ps})$ & $\mathrm{B}$ & $\mathrm{T}_{0}\left({ }^{\circ} \mathrm{C}\right)$ & $\mathrm{d} \log (\tau) / \mathrm{d}\left(\mathrm{T}_{\alpha} / \mathrm{T}\right)$ at $\mathrm{T}=\mathrm{T}_{\alpha}$ \\
\hline PS & atactic polystyrene & 0.019 & 0.48 & 3.04 & 1152 & 64.4 & 101 \\
PS3VP & 97\% styrene-3\% vinylphenol & 0.064 & 0.44 & 1.87 & 1194 & 64.5 & 101 \\
PS7VP & $93 \%$ styrene-7\% vinylphenol & 0.114 & 0.43 & 0.375 & 1377 & 62.7 & 99 \\
PS10VP & $90 \%$ styrene-10\% vinylphenol & 0.175 & 0.42 & 0.285 & 1425 & 64.4 & 98 \\
PS18VP & 82\% styrene-18\% vinylphenol & 0.078 & 0.41 & $9.68 \times 10^{-4}$ & 2188 & 51.7 & 92
\end{tabular}

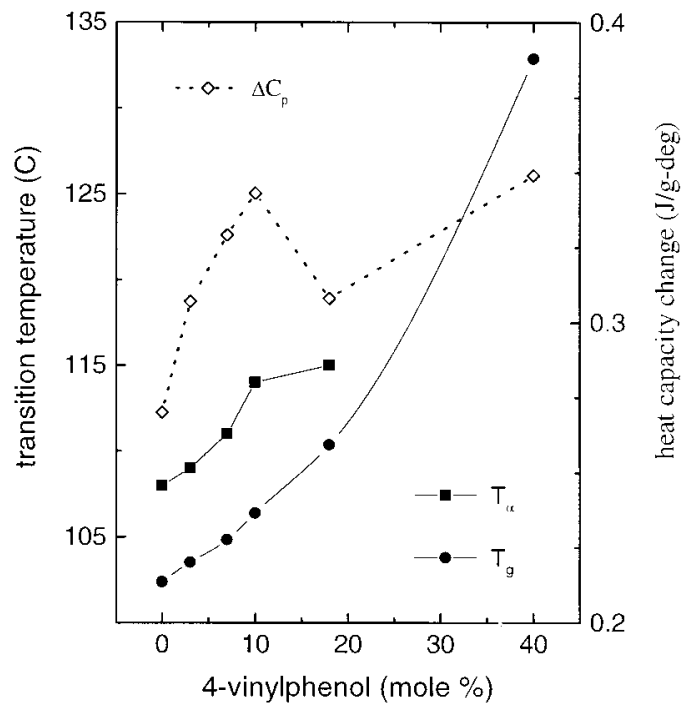

Figure 3. Dependence on chemical structure of the glass transition temperature $(\bullet)$ and the temperature at which $\tau_{\alpha}$ $=1 \mathrm{~s}(\boldsymbol{\square})$. Also shown is the heat capacity increment at $\mathrm{T}_{\mathrm{g}}$ measured by DSC $(\diamond)$.

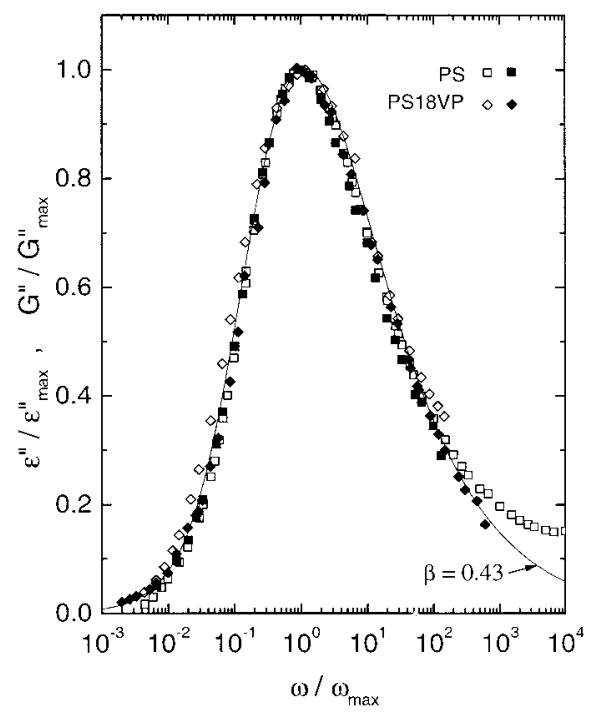

Figure 4. Dispersion in the dielectric loss (hollow symbols) and the loss modulus (solid symbols). The data have been shifted to superimpose the peaks. The solid line is the KWW function (eq 5).

spectra exhibit this same positive deviation from the KWW function. $35,37,42,43$

The breadth of the response function, $\phi(\mathrm{t})$ (or its degree nonexponentiality, as characterized by $\beta$ ), for both polymers and small molecule glass formers is known to correlate with the degree to which the temperature dependence deviates from Arrhenius behavior . ${ }^{7,8,13,44-50}$ Only a few exceptions to this trend have been reported, e.g., low molecular weight polystyrene ${ }^{28}$ and propylene carbonate. ${ }^{49}$ The normalized temperature dependence, or fragility, is defined as the slope of the quantity $\mathrm{d} \log (\tau) / \mathrm{d}\left(\mathrm{T}_{\alpha} / \mathrm{T}\right)$ evaluated at $\mathrm{T}_{\alpha}$. In terms of

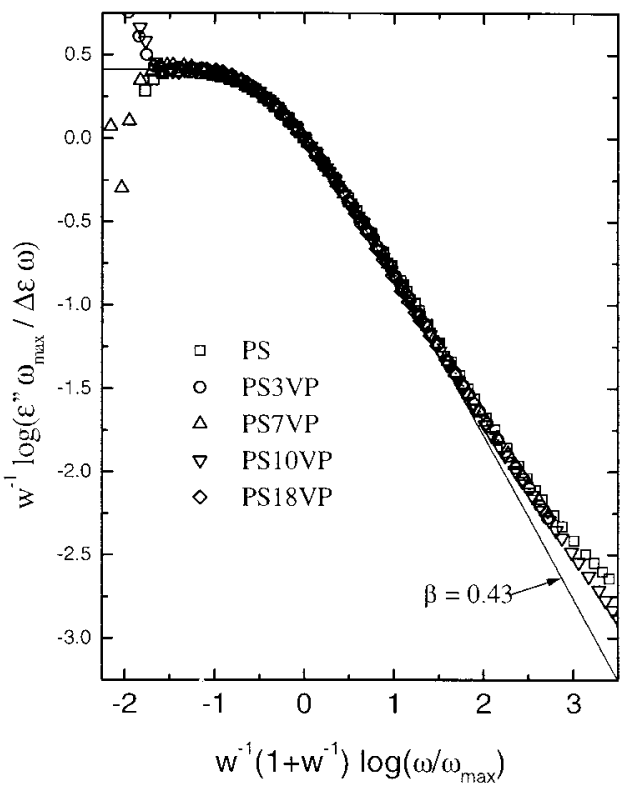

Figure 5. Dielectric data scal ed according to the proposal of Dixon and Nagel. ${ }^{35-38}$ The solid line is the KWW function (eq $5)$.

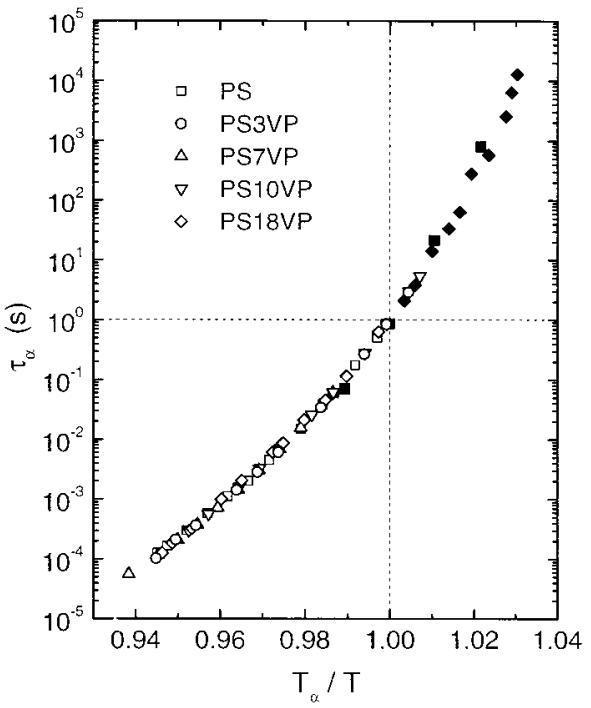

Figure 6. Fragility curves for the dielectric (hollow symbols) and mechanical (solid symbols) relaxation times.

the Vogel-Fulcher parameters, fragility can be expressed $\mathrm{as}^{51}$

$$
\left.\frac{\mathrm{d} \log (\tau)}{\mathrm{d}\left(\mathrm{T}_{\alpha} / \mathrm{T}\right)}\right|_{\mathrm{T}=\mathrm{T}_{\alpha}}=\frac{\mathrm{b}}{\ln (10) \mathrm{T}_{\alpha}}\left(1-\mathrm{T}_{\infty} / \mathrm{T}_{\alpha}\right)^{-2}
$$

Figure 6 shows fragility plots for the polymers. Consistent with the comparable breadths of their relaxation functions, the temperature dependences are equivalent.

Different results can be obtained when different experimental methods are used to characterize segmental relaxation. For example, it is well-known that 
mechanical relaxation times are invariably shorter than $\tau_{\alpha}$ measured dielectrically. $29,52-54$ In some cases, even the shape of the relaxation function (i.e., the $\beta$ in eq 5), as well as the temperature dependence of $\tau_{\alpha}$, can depend on the experimental probe.55 In Figure 2 we show mechanical data for the PS 28 and for PS18VP. These mechanical relaxation times are shorter than the corresponding $\tau$ measured dielectrically.

Although the shapes of the curves for the mechanical data in Figure 2 are similar to those for dielectric measurements, the respective temperature dependences are best compared using the fragility scheme. Mechanical results have been included in Figure 6, revealing that the temperature dependences as measured by the two spectroscopies are the same within experimental error. This being the case, we expect the breadth of the segmental dispersion for the loss modulus to be similar to that for the dielectric loss. This expectation is borne out by the data shown in Figure 4. Thus, mechanical spectroscopy corroborates the invariance of the relaxation behavior to polymer composition, as observed dielectrically.

\section{Summary}

Broadening of the segmental relaxation function has a well-established cause. For polymers and glassforming liquids in dense phase, there exist intermolecular constraints on segmental relaxation, in the form of density fluctuations, steric interferences, etc., from the local environment. These constraints give rise to intermolecular cooperativity (many-body dynamics), which slow the relaxation rate and broaden the dispersion. $4,42,56-60$ For polymers, intramolecular cooperativity due to the chain connectivity may also contribute to the nonexponentiality of the relaxation. ${ }^{61,62}$

There is another broadening mechanism that is unique to random copolymers. It has also been shown that chemical heterogeneity of the chain backbone can broaden the $\alpha$-dispersion. The effect has been attributed to a distribution of relaxation behaviors, corresponding to different conformational transition rates (e.g., HallHelfand type motion ${ }^{63}$ ) for the various backbone units. Such inhomogeneous broadening has been reported for copolymers of 2-methyl-1,2-epoxy-1,4-butanediyl and 2-methyl-1-butenylene (epoxidized natural rubber) ${ }^{8}$ and ethylene-propylene copolymers. ${ }^{12}$ H owever, the effect is negligible in the present case, presumably because the addition of a hydroxyl group to the pendant phenyl ring does not directly involve the backbone bonds.

The invariance to chemical structure of both the spectral breadth and the temperature dependence of $\tau_{\alpha}$ indicates there is no variation among these polymers in the degree of intermolecular cooperativity associated with their segmental relaxation. Due to the modest structural difference between styrene and vinylphenol repeat units, there is little enhancement of steric constraints on the dynamics. Polar interactions involving the phenol group are certainly present; however, their lifetime is evidently too short relative to the time scale for segmental relaxation. Consequently, they effect only an increased mean-field, frictional resistance. Sustained interactions, in the manner of permanent cross-links, would modify the degree of cooperativity of the motion, with changes in both the relaxation function and the fragility. ${ }^{64,65}$ The polar interactions herein exert only a viscous drag. In this manner, the relaxation times increase (as does $T_{g}$ ), without change in either the relaxation rate (i.e., relaxation function) or its temperature dependence. In the language of the coupling model, ${ }^{5,59}$ chemical modification of the polystyrene alters the magnitude of the noncooperative (primitive) relaxation time, without changing the coupling strength.

It is noteworthy that while fragility is essentially independent of chemical structure for these materials (Table 1), the heat capacity increment at $\mathrm{T}_{\mathrm{g}}$ is an increasing function of the vinylphenol level. This trend is similar to data on poly(dimethylsiloxane)s, in which increases in molecular weight reduced $\Delta C_{p}\left(T_{q}\right)$ without affecting fragility. Such results are at odds with Angell's energy landscape model, which predicts that larger $\Delta \mathrm{C}_{\mathrm{p}}$ $\left(T_{g}\right)$ is associated with more fragile behavior. $27,50,66$ Although Angell's model has enjoyed some success in accounting for the effect of temperature on the viscosity of small-molecule glass formers, $27,66,67$ as well as computer simulations of Leonard-J ones fluids, ${ }^{68}$ the behavior of polymers, ${ }^{69}$ including polystyrene ${ }^{28,70}$ and polyvinylethylene networks, ${ }^{71}$ is not satisfactorily addressed. Approaches that focus directly on cooperativity and the dynamical constraints inherent to this many-body problem may prove more fruitful.

Acknowledgment. This work was supported by the Office of Naval Research, M.J.S., through the Naval Academy Research Council (ONR Grant N0001496WR20008). We thank E. M. Pearce for assisting with synthesis of the copolymers.

\section{References and Notes}

(1) The 2nd International Meeting on Relaxations in Complex Systems, Alicante, Spain, 1994. J . Non-Cryst. Solids 1994, 172 .

(2) The 3rd International Meeting on Relaxations in Complex Systems, Vigo, Spain, 1997. J . Non-Cryst. Solids 1998, 235.

(3) The 1st International Meeting on Relaxations in Complex Systems, Crete, Greece, 1991. J. Non-Cryst. Sol ids 1991, 131.

(4) Adam, G.; Gibbs, J . H. J . Chem. Phys. 1965, 43, 139.

(5) Ngai, K. L.; Rendell, R. W.; Rajagopal, A. K.; Teitler, S. Ann. N.Y. Acad. Sci. 1986, 484, 150.

(6) Matsuoka, S. Relaxation Phenomena in Polymers; Hanser: New York, 1992.

(7) Roland, C. M.; Ngai, K. L. Macromolecules 1991, 24, 5315; 1992, 25, 1844.

(8) Roland, C. M. Macromolecules 1992, 25, 7031.

(9) Zorn, R.; McKenna, G. B.; Willner, L.; Richter, D. Macromolecules 1995, 28, 8552.

(10) Alvarez, F.; Colmenero, J .; Wang, C. H.; Zia, J . L.; Fytas, G. Macromolecules 1995, 28, 6488.

(11) Fitzgerald, J .; Binga, T. D.; Sorriero, L. J .; O'Reilly, J . M. Macromolecules 1995, 28, 7401.

(12) Santangelo, P. G.; Ngai, K. L.; Roland, C. M. Macromolecules 1996, 29, 3651.

(13) Hofmann, A.; Alegria, A.; Colmenero, J .; Willner, L.; Buscaglia, E.; Hadjichristidis, N. Macromol ecules 1996, 29, 129.

(14) Simon, G. P.; Beatty, C. L.; Andrews, S. R.; Shinton, S.; Williams, G. Polymer 1998, 24, 5929.

(15) Andreis, M.; Rakvin, B.; Veksli, Z.; Rogosic, M.; Mencer, H. J . Polymer 1999, 40, 1955.

(16) Chen, C.-T.; Morawetz, H. Macromolecules 1989, 22, 159.

(17) J ong, L.; Pearce, E. M.; Kwei, T. K.; Dickinson, L. C. Macromolecules 1990, 23, 5071.

(18) J ong, L.; Pearce, E. M.; Kwei, T. K. Polymer 1993, 34, 48.

(19) Zhuang, H. F.; Pearce, E. M.; Kwei, T. K. Macromolecules 1994, 27, 6398.

(20) Landry, M. R.; Massa, D. J .; Landry, C. J . T.; Teeharden, D. M.; Colby, R. H.; Long, T. E.; Henrichs, P. M. J . Appl. Polym Sci. 1994, 54, 991.

(21) Xiang, M.-L.; J iang, M.; Zhang, Y.; Wu, C.; Feng, L. Macromolecules 1997, 30, 2313.

(22) Li, L.; Chan, C.-M.; Weng, L.-T.; Xiang, M.-L.; Jiang, M. Macromolecules 1998, 31, 7248. 
(23) Du, Y.; Xue, Y.; Frisch, H. L. In Physical Properties of Polymers Handbook; Mark, J. E., Ed.; American Institute Physics Press: Woodbury, NY, 1996; Chapter 16.

(24) Mott, N. F.; Davis, E. A. Electronic Processes in NonCrystalline Materials; Clarendon Press: Oxford, UK, 1979.

(25) Boese, D.; Kremer, F. Macromolecules 1990, 23, 829.

(26) Ferry, J . D. Viscoelastic Properties of Polymers; Wiley: New York, 1980.

(27) Angell, C. A. J . Non-Cryst. Solids 1991, 131-133, 13.

(28) Santangelo, P. G.; Roland, C. M. Macromolecules 1998, 31, 4581.

(29) McCrum, N. G.; Read, B. E.; Williams, G. Anelastic and Dielectric Effects in Polymer Solids; Wiley: London, 1967.

(30) Alexandrovich, P. S.; Karasz, F. E.; MacKnight, W. J . Polymer 1980, 21, 488.

(31) Rellick, G. S.; Runt, J .J . Polym. Sci., Polym. Phys. Ed. 1986, 24, 313

(32) Sanchis, M. J .; Martinez, E. S.; Calleja, R. D.; Pankratova, E. T.; Murin, I. Polym. Int. 1996, 41, 337.

(33) Kohlrausch, R. . Ann. Phys. (Leepzig) 1847, 12, 393.

(34) Williams, G.; Watts, D. C. Trans. Faraday Soc. 1970, 66, 80.

(35) Dixon, P. K.; Wu, L.; Nagel, S. R.; Williams, B. D.; Carini, J . P. Phys. Rev. Lett. 1990, 65, 1108.

(36) Wu, L.; Dixon, P. K.; Nagel, S. R.; Williams, B. D.; Carini, J . P. J . Non-Cryst. Solids 1991, 131-133, 32.

(37) Menon, N.; O'Brien, K. P.; Dixon, P. K.; Wu, L.; Nagel, S. R. J. Non-Cryst. Solids 1992, 141, 61.

(38) Leheny, R. L.; Nagel, S. R. J . Non-Cryst. Solids 1998, 235237, 278.

(39) Schönhals, A.; Kremer, F.; Hofmann, A.; Fischer, E. W.; Schlosser, E. Phys. Rev. Lett. 1993, 70, 3459.

(40) Kudlik, A.; Benkhof, S.; Lenk, R.; Rössler, E. Eur. Lett. 1995 $32,511$.

(41) Dixon, P. K. Phys. Rev. Lett. 1990, 42, 8179

(42) Matsuoka, S.; Quan, X. Macromol ecules 1991, 24, 2770.

(43) León, C.; Ngai, K. L.; Roland, C. M.J . Chem. Phys., in press.

(44) Plazek, D. J .; N gai, K. L. Macromolecules 1991, 24, 1222.

(45) Roland, C. M.; Ngai, K. L. Macromolecules 1992, 25, 5765.

(46) Roland, C. M.; Ngai, K. L. Macromolecules 1992, 25, 363.

(47) Ngai, K. L.; Roland, C. M.; O'Reilly, J. M.; Sedita, J. S. Macromol ecules 1992, 25, 3906.
(48) Ngai, K. L.; Roland, C. M. Macromolecules 1993, 26, 6824.

(49) Bohmer, R.; Ngai, K. L.; Angell, C. A.; Plazek, D. J . J . Chem. Phys. 1993, 99, 4201.

(50) Angell, C. A. Science 1995, 267, 1924.

(51) Hodge, I. M. J . Non-Cryst. Solids 1996, 202, 164

(52) Santangelo, P. G.; Roland, C. M. Macromolecules 1998, 31, 3715.

(53) Adachi, K.; Yoshida, H.; Fukui, F.; Kotaka, T. Macromol ecules 1990, 23, 3138

(54) Colmenero, J.; Alegria, A.; Santangelo, P. G.; Ngai, K. L.; Roland, C. M. Macromolecules 1994, 27, 407.

(55) Ngai, K. L.; Mashimo, S.; Fytas, G. Macromolecules 1988 $21,3030$.

(56) Tobolsky, A. V.; DuPre, D. B. Adv. Polym. Sci. 1969, 6, 103.

(57) Schönhals, A.; Schlosser, E. Colloid Polym. Sci. 1989, 267, 125.

(58) Götze, W.; Sjögren, L. Rep. Prog. Phys. 1992, 55, 241.

(59) Ngai K. L.; Rendell R. W. J . Non-Cryst. Solids 1991, 131$133,942$.

(60) Ngai, K. L. J . Phys.: Condens. Matter 1999, 11, 1.

(61) Bahar, I.; Erman, B.; Kremer, F.; Fischer, E. W. Macromolecules 1992, 25, 816

(62) Bahar, I.; Erman, B.; Fytas, G.; Steffen, W. Macromolecules 1994, 27, 5200.

(63) Hall, C. K.; Helfand, E. J . Chem. Phys. 1982, 77, 3275.

(64) Roland, C. M.; Ngai, K. L.; Plazek, D. J . J . Comput. Theor. Polym. Sci. 1997, 7, 133.

(65) Roland, C. M. Macromolecules 1994, 27, 4242.

(66) Angell, C. A. Nature 1998, 393, 521.

(67) Angell, C. A. In Relaxations in Complex Systems; Ngai, K. L., Wright, G. B., Eds.; Government Printing Office: Washington, DC, 1985; p 3.

(68) Sastry, S.; Debenedetti, P. G.; Stillinger, F. H. Nature 1998, 393, 554.

(69) Roland, C. M.; Santangelo, P. G.; Ngai, K. L. J . Chem. Phys., in press.

(70) Roland, C. M.; Santangelo, P. G.; Antonietti, M.; Neese, M. Macromolecules 1999, 32, 2283.

(71) Santangelo, P. G.; Roland, C. M. Phys. Rev. B 1998, 58, 14121. MA990317N 